Core 7 and allow division of the Coccolithus doronicoides Zone into lower and upper subzones at DSDP 132.

\section{Coccolithus doronicoides Zone}

\section{Gephyrocapsa caribbeanica Subzone}

The assemblage of the upper subzone of the $C$. doronicoides Zone is similar to that of the $E$. annula Subzone, but the first appearance and common occurrence of Gephyrocapsa caribbeanica below the first appearance of $G$. oceanica is used to define this unit.

\section{Gephyrocapsa oceanica Zone}

This unit is defined at the base by the first occurrence of $G$. oceanica. Assemblages of the zone are characterized by Ceratolithus cristatus, Cyclococcolithina leptopora, Gephyrocapsa aperta, G. caribbeanica, G. oceanica, Helicopontosphaera kamptneri [H. sellii absent or rare], Rhabdosphaera clavigera, and Scapholithus sp. The top of this unit is based, in light-microscope study, on the occurrence above of a great abundance of Emiliania huxleyi, a small (2 to 3 micron) faint oval coccolith.

The Pleistocene zonal assemblages of DSDP 132, in this and adjacent zones, are distinguished from open-ocean assemblages by the presence throughout of Braarudosphaera bigelowi, a marginal-marine species, and by the absence of Ceratolithus cristatus, a fully marine species. Gephyrocapsa caribbeanica, the most temperature-tolerant species of Gephyrocapsa, occurs commonly through the Pleistocene cores. The warm-water species $G$. oceanica occurs most commonly at the top of Core 7, considered the lower part of the Gephyrocapsa oceanica Zone. Occurrences in higher cores are sparse and discontinuous.

\section{Emiliania huxleyi Zone}

This zone, the highest coccolith zone recognized, is characterized by the overwhelming dominance of Emiliania huxleyi. This species cannot be definitely identified by light microscopy, but dominance of small coccoliths at the top of oceanic-sediment sections in conjunction with narrowrimmed specimens of Helicopontosphaera kamptneri gen- erally results in the identification of $E$. huxleyi when electron-microscope study is carried out.

Sample $132-1-1,142$ to $143 \mathrm{~cm}$ contains abundant ?Emiliania huxleyi and some small, narrow-rimmed Helicopontosphaera kamptneri, with Coccolithus pelagicus, Cyclococcolithina leptopora, and Gephyrocapsa sp. cf. $G$. caribbeanica.

\section{REFERENCES}

Boudreaux, J. E. and Hay, W. W., 1969. Calcareous nannoplankton and biostratigraphy of the late PliocenePleistocene-Recent sediments in the Submarex cores. Revista Espanola de Micropaleontologia. 1, 249.

Bramlette, M. N. and Riedel, W. R., 1954. Stratigraphic value of discoasters and some other microfossils related to recent coccolithophores. J. Paleontology. 28, 403.

Bukry, D., 1971. Coccolith stratigraphy Leg 7, Deep Sea Drilling Project. Initial Reports of the Deep Sea Drilling Project, Volume VII. Washington (U.S. Government Printing Office).

Bukry, D. and Bramlette, M. N., 1970. Coccolith age determinations Leg 3, Deep Sea Drilling Project. Initial Reports of the Deep Sea Drilling Project, Volume III. Washington (U.S. Government Printing Office). 589.

Gartner, S., Jr., 1969. Correlation of Neogene planktonic foraminifer and calcareous nannofossil zones. Gulf Coast Assoc. Geol. Soc. Trans. 19, 585.

1970. Coccolith age determinations Leg 3, Deep Sea Drilling Project. Initial Reports of the Deep Sea Drilling Project, Volume III. Washington (U.S. Government Printing Office). 613.

Hay, W. W., Mohler, H., Roth, P. H., Schmidt, R. R. and Boudreaux, J. E., 1967. Calcareous nannoplankton zonation of the Cenozoic of the Gulf Coast and Caribbean-Antillean area, and transoceanic correlation. Gulf Coast Assoc. Geol. Soc. Trans. 17, 428.

Martini, E. and Bramlette, M. N., 1963. Calcareous nannoplankton from the experimental Mohole drilling. $J$. Paleontology. 37, 845.

Riedel, W. R., Bramlette, M. N. and Parker, F. L., 1963. "Pliocene-Pleistocene" boundary in deep-sea sediments. Science. 140, 1238.

\title{
33.2. CALCAREOUS NANNOFOSSIL AGE DETERMINATIONS, DEEP SEA DRILLING PROJECT, LEG 13
}

\author{
Stefan Gartner, Jr., Rosenstiel School of Marine and Atmospheric Science, \\ University of Miami, Miami, Florida
}

This paper contains age determinations made on a suite of samples from the cores collected during Leg 13 of the Deep Sea Drilling Project. With the exception of Site 120 in the eastern North Atlantic, all samples containing nannofossils are Neogene in age. The samples from Site 120 were dated approximately, based on meager literature available for the Jurassic and lower Cretaceous calcareous nannofossils. Inconsistency in the results obtained by the several workers who have studied this interval, lack of rigorous nomenclature, and the absence of nannoconids in the samples, make the ages assigned to Samples 13-120-2-1; 70 $\mathrm{cm}$ and 13-120-7-1;33-34 $\mathrm{cm}$ mere estimates. 
The age assignments for the remaining samples, for the most part, can be considered fairly reliable despite frequent contamination of Neogene suites by older Tertiary and Cretaceous coccoliths. The zonation scheme used here for the Pliocene-Pleistocene is that proposed by Gartner (1969) and subsequently incorporated by Martini and Worsley (Figure 1). The Discoaster pentaradiatus Zone, which was added by Martini and Worsley (1970) and was indicated as an identifiable interval in the range chart of Gartner (1969) is not used here. The time interval represented by this zone is so short as to make it of no practical use in deep-sea sediment.

In some instances samples were not dated as to zone, instead broader age assignments are indicated. These samples generally contain a provincial nannofossil suite with key species lacking; or the assemblage is mixed over a short interval but across zonal boundaries so that mutually exclusive species may occur together.

\begin{tabular}{|c|c|}
\hline Pleistocene & $\begin{array}{l}\text { Emiliania huxleyi } \\
\text { Gephyrocapsa oceanica } \\
\text { Pseudoemiliania lacunosa }\end{array}$ \\
\hline Pliocene & $\begin{array}{l}\text { Discoaster brouweri } \\
\text { Discoaster surculus } \\
\text { Reticulofenestra pseudoumbilica } \\
\text { Discoaster asymmetricus } \\
\text { Ceratolithus rugosus }\end{array}$ \\
\hline Miocene & $\begin{array}{l}\text { Ceratolithus tricorniculatus } \\
\text { Discoaster quinqueramus }\end{array}$ \\
\hline
\end{tabular}

Figure 1. Neogene calcareous nannofossil zones.

13-120-2-1, $70 \mathrm{~cm}$ :

Maslovella barnesae, Cretarhabdus, sp., Lithraphidites carniolensis (no Prediscosphaera; no Eiffellithus), Cyclagelosphera margerelli, Staurolithites sp., Markalius circumradiatus, Micrantholithus hochschulzi cf. Calcicalathina oblonga, Rucinolithus irregularis Corollithion exiguum, cf. Cretaturbella rothii, Cruciellipsis cuvillieri.

Age: Late Valanginian to early Hauterivian.

13-120-7-1, 33-44 cm:

Diazomatolithus lehmani, Cretarhabdus conicus, Cruciellipsis cuvillieri, Coccolithus britannicus, Stephanolithion laffittei, Podorhabdus sp.

Age: ? Portlandian - Purbeckian.

13-121-1-1, 17-18 cm:

Coccolithus pelagicus, Gephyrocapsa oceanica

Age: Gephyrocapsa oceanica Zone.

13-121-1-5, 0-1 cm:

Age: As above.

13-121-2-1, 4-5 cm:

Age: As above.

13-121-2-6, 3-4 cm:

Age: As above.

13-121-3-1, 102-103 cm:

Gephyrocapsa oceanica, Pseudoemiliania lacunosa.

Age: Pseudoemiliania lacunosa Zone.

13-121-3-4, 9-10 cm:

Age: As above.
13-121-4-1, 2-3 cm:

Age: As above.

13-121-4-6, 3-4 cm:

Age: As above.

13-121-7-1, 105-106 cm:

Age: As below.

13-121-8-1:

Pseudoemiliania lacunosa, Cyclococcolithina macintyrei (no discoasters).

Age: Probably Discoaster brouweri Zone, late Pliocene.

13-121-9-1, 95-96 cm:

Discoaster sp. (6 rays, possibly indigenous).

Age: As above.

13-121-10-1, 74-75 cm:

Discoaster brouweri, cf. Discoaster surculus, Pseudoemiliania lacunosa.

Age: Discoaster surculus Zone.

13-121-10-2, 12-13 cm:

Age: As above.

13-121-13-1, 133-134 cm:

Age: As above.

13-121-14-1, 120-121 cm:

Reticulofenestra pseudoumbilica, Pseudoemiliania lacunosa.

Age: Probably as above.

13-121-15-1, pieces:

Calcareous detritus; no nannofossils.

13-121-19-1, 120-122 cm:

Discoaster variabilis, Reticulofenestra pseudoumbilica .

Age: Probably pre-Reticulofenestra pseudoumbilica Zone.

13-121-22-CC, 116-117 cm:

Sphenolithus abies; Reticulofenestra pseudoumbilica .

Age: Late Neogene.

13-121-23-2, $87-88 \mathrm{~cm}$ :

Age: As above.

13-121-24-1, 114-117 cm:

Discoaster variabilis, Discoaster exilis, Sphenolithus abies, Reticulofenestra pseudoumbilica.

Age: Neogene, late Miocene to early Pliocene.

13-121-24-2, 93-94 cm:

Discoaster exilis, Reticulofenestra pseudoumbilica, Sphenolithus abies, Discoaster variabilis, cf. Discoaster surculus.

Age: Late Miocene to early Pliocene.

13-122-1-1, 107-108 cm:

Emiliania huxleyi, Gephyrocapsa spp.

Age: Emiliania huxleyi Zone.

13-122-1-2, 3-4 cm:

Gephyrocapsa spp.

Age: Gephyrocapsa oceanica Zone.

13-122-CC, $2-3 \mathrm{~cm}$ :

Reticulofenestra pseudoumbilica, Sphenolithus abies.

Age: Pre-mid Pliocene.

13-122-2-2, 9-10 cm:

Age: Probably as above. 
13-122-3-CC, $1-2 \mathrm{~cm}$ :

Calcareous detritus, no nannofossils.

13-123-1-2, 2-3 cm:

Emiliania huxleyi.

Age: Emiliania huxleyi Zone.

13-123-1-3, 2-3 cm:

Calcareous detritus; no nannofossils.

13-123-2-1, 77-78 cm:

Pseudoemiliania lacunosa, Gephyrocapsa oceanica.

Age: Pseudoemiliania lacunosa Zone.

13-123-3-1, 88-89 cm:

Pseudoemiliania lacunosa, Discoaster pentaradiatus, Cocco-

lithus pelagicus.

Age: Late Pliocene.

13-123-4-2, $17-20 \mathrm{~cm}$ :

Age: As above.

13-123-4-3, 15-16 cm:

Age: As above.

13-123-5-1, 97-98 cm:

Discoaster tamalis, Discoaster asymmetricus, Sphenolithus abies, Reticulofenestra pseudoumbilica.

Age: Early to mid Pliocene.

13-123-5-2, 1-2 cm:

Age: As above.

13-123-6-1, 83-84 cm:

Age: As above.

13-124-1-1, 2-3 cm:

Pseudoemiliania lacunosa, Gephyrocapsa oceanica.

Age: Pseudoemiliania lacunosa Zone.

13-124-1-6, 1-2 cm:

Age: As above.

13-124-2-1, 26-27 cm:

Pseudoemiliania lacunosa, Cyclococcolithina macintyrei, Coccolithus pelagicus (no Gephyrocapsa).

Age: Probably latest Pliocene.

13-124-2-4, 1-2 cm:

Age: As above.

13-124-3-1, 105-106 cm:

Reticulofenestra pseudoumbilica, Discoaster asymmetricus, Discoaster brouweri.

Age: Early to mid Pliocene.

13-124-3-2, 5-6 cm:

Age: As above.

13-124-4-1, 81-82 cm:

Age: Probably as above.

13-124-4-4, 1-2 cm:

Discoaster surculus, Reticulofenestra pseudoumbilica, Discoaster pentaradiatus, Sphenolithus abies.

Age: Probably late Miocene.

13-124-5-1, 102-104 cm:

Age: As above.

13-124-5-2, 8-9 cm:

Age: As above.
13-124-7-1, 1-2 cm:

Mostly detritus, insufficient material, poor preservation.

13-124-10-2, 99-100 cm:

No calcareous nannofossils.

13-125-1-1, $10 \mathrm{~cm}$ :

Gephyrocapsa spp.

Age: Gephyrocapsa oceanica Zone.

13-125-1-5, $15 \mathrm{~cm}$ :

Age: As above.

13-125-2-1, $120 \mathrm{~cm}$ :

Pseudoemiliania lacunosa, Gephyrocapsa spp.

Age: Pseudoemiliania lacunosa Zone.

13-125-2-5, $70 \mathrm{~cm}$ :

Age: As above.

13-125-3-1, $44-45 \mathrm{~cm}$ :

Age: As above.

13-125-3-6, 3-4 cm:

Age: As above.

13-125-4-1, 10-11 cm:

Age: As above.

13-125-4-6, 10-11 cm:

Pseudoemiliania lacunosa (no Gephyrocapsa, no Discoaster). Age: Probably latest Pliocene.

13-125-5-1, 10-11 cm:

Age: As above.

13-125-5-CC, 70-71 cm:

Discoaster pentaradiatus, Pseudoemiliania lacunosa, Coccolithus pelagicus, Cyclococcolithina leptopora, Discoaster surculus.

Age: Discoaster surculus Zone.

13-125-6-1, 10-11 cm:

Age: As above.

13-125-6-3, 13-14 cm:

Age: As above.

13-125-7-2, 10-11 cm:

Age: As above.

13-125-7-5, 10-11 cm:

Age: As above.

13-125A-1-1, 74-75 cm:

Cyclococcolithina macintyrei, Pseudoemiliania lacunosa.

Age: Probably late Pliocene.

13-125A-1-2, 10-11 cm:

Discoaster brouweri, Pseudoemiliania lacunosa.

Age: Discoaster brouweri Zone.

13-125A-1-2, 2-3 cm:

Reticulofenestra pseudoumbilica, Pseudoemiliania lacunosa; mixed assemblage.

13-125A-2-1, $70-71 \mathrm{~cm}$ :

Pseudoemiliania lacunosa, Cyclococcolithina macintyrei. Age: Late Pliocene.

13-125A-3-1, 30-31 cm:

Age: As above. 
13-125A-4-1, 7-8 cm:

Discoaster tamalis (no Pseudoemiliania lacunosa, no Reticulofenestra pseudoumbilica).

Age: Mid Pliocene.

13-125A-4-2, 86-87 cm:

Age: As above.

13-125A-5-1, 101-102 cm:

Age: Probably as above.

13-125A-5-4, 4-5 cm:

Sphenolithus abies, Discoaster surculus, Reticulofenestra pseudoumbilica, Discoaster pentaradiatus.

Age: Early Pliocene.

13-126-1-2:

Gephyrocapsa spp.

Age: Probably Gephyrocapsa oceanica Zone.

13-126-1-6, 11-12 cm:

Gephyrocapsa spp., Pseudoemiliania lacunosa.

Age: Pseudoemiliania lacunosa Zone.

13-126-2-1, 16-17 cm:

Age: As above.

13-126-2-5, 113-114 cm:

Age: As above.

13-126-3-1, $134 \mathrm{~cm}$ :

Age: As above.

13-126-3-2, 10-11 cm:

Age: As above.

13-126-6-1, $100 \mathrm{~cm}$ :

Reticulofenestra pseudoumbilica .

Age: Mid-Miocene to mid-Pliocene (inadequate assemblage).

13-126A-1-1, 125-126 cm:

Discoaster variabilis, Reticulofenestra pseudoumbilica, Discoaster aff. D. bollii.

Age: Probably late middle Miocene.

13-126A-1-1, $135 \mathrm{~cm}$ :

Age: As above.

13-127-1-1, 13-14 cm:

Gephyrocapsa spp.--mixed assemblage.

Age: Pleistocene, probably late Pleistocene.

13-127-1-5, 13-14 cm:

Age: As above.

13-127-2-1, 70-71 cm:

?Emiliania huxleyi, Gephyrocapsa spp.

Age: Probably as above.

13-127-2-5, 10-11 cm:

Gephyrocapsa spp.

Age: Probably Gephyrocapsa oceanica Zone.

13-127-4-2, 14-15 cm:

Age: As above.

13-127-5-2, 10-11 cm:

Age: As above.

13-127-6-2, 10-11 cm:

Pseudoemiliania lacunosa, Gephyrocapsa spp.

Age: Pseudoemiliania lacunosa Zone.
13-127-7-2, 10-11 cm:

Age: As above.

13-127-8-2, 10-11 cm:

Age: As above.

13-127-9-1, 30-31 cm:

Age: As above.

13-127-10-2, 10-11 cm:

Age: As above.

13-127-11-1, 85-86 cm:

Age: As above.

13-127-12-3, 10-11 cm:

Age: As above.

13-127-13-1, 49-50 cm:

Age: As above.

13-127-14-1, 10-11 cm:

Age: As above.

13-127-14-6, 11-12 cm:

Age: As above.

13-127-15-2, 10-11 cm:

Age: As above.

13-127-18-1, 79-80 cm:

Pseudoemiliania lacunosa, Discoaster brouweri.

Age: Discoaster brouweri Zone.

13-127A-2-2, 10-11 cm:

Pseudoemiliania lacunosa, Gephyrocapsa spp., Emiliania huxleyi.

Age: Mixed Pleistocene assemblage.

13-127A-2-3, 10-11 cm:

Gephyrocapsa spp., Pseudoemiliania lacunosa.

Age: Pseudoemiliania lacunosa Zone.

13-127A-3-1, 125-126 cm:

Age: As above.

13-127A-3-6, 10-11 cm:

Age: As above.

13-127A-4-2, 10-11 cm:

Age: As above.

13-127A-4-5, 6-7 cm:

Age: As above.

13-128-1-1, 140-141 cm:

Emiliania huxleyi.

Age: Emiliania huxleyi Zone.

13-128-1-3, $37 \mathrm{~cm}$ :

Age: Gephyrocapsa oceanica Zone.

13-128-2-2, 10-11 cm:

Pseudoemiliania lacunosa, Gephyrocapsa spp.

Age: Pseudoemiliania lacunosa Zone.

13-128-3-3, 59-60 cm:

Age: As above.

13-128-4-2, 10-11 cm:

Age: As above.

13-128-5-2, 10-11 cm:

Age: As above. 
13-128-6-2, $10 \mathrm{~cm}$ :

Age: As above.

13-128-7-2, 13-14 cm:

Age: As above.

13-128-8-2, $10 \mathrm{~cm}$ :

Age: As above.

13-128-10-2, $25 \mathrm{~cm}$ :

Age: As above.

13-128-11-2, 13-14 cm:

Age: As above.

13-129-2-1, 87-89 cm:

Calcareous detritus with mixed Neogene nannofossil assemblage.

13-129-3-1, 15-16 cm:

Reticulofenestra pseudoumbilica, Sphenolithus abies, Discoaster cf. D. exilis (contaminated).

Age: Probably late middle Miocene.

13-129A-3-1, $15 \mathrm{~cm}$ :

Reticulofenestra pseudoumbilica, Sphenolithus belemnos, Sphenolithus abies (mixed assemblage).

Age: Possibly somewhere in the middle Miocene.

13-130-1-1, $100 \mathrm{~cm}$ :

Pseudoemiliania lacunosa, Gephyrocapsa spp.

Age: Pseudoemiliania lacunosa Zone.

13-130-1-2, $5 \mathrm{~cm}$ :

Age: As above.

13-130-1-4, 12-13 cm:

Age: As above.

13-130-2-1, $46 \mathrm{~cm}$ :

Age: As above.

13-130-2-3, $10 \mathrm{~cm}$ :

Age: As above.

13-130-3-2, $28 \mathrm{~cm}$ :

Age: As above.

13-130-4-2, $7 \mathrm{~cm}$ :

Age: As above.

13-130-5-2, $15 \mathrm{~cm}$ :

Pseudoemiliania lacunosa (sparse assemblage).

Age: Mid Pliocene to Pleistocene.

13-130-5-3, 11-12 cm:

Pseudoemiliania lacunosa, Gephyrocapsa spp.

Age: Pseudoemiliania lacunosa Zone.

13-130-6-2, $74 \mathrm{~cm}$ :

Pseudoemiliania lacunosa, Discoaster brouweri.

Age: Discoaster brouweri Zone.

13-130-6-4, 50-51 cm:

Age: As above.

13-131-1-1, 10-11 cm:

Gephyrocapsa spp.

Age: Probably Gephyrocapsa oceanica Zone.

13-131 A-1-1, $137 \mathrm{~cm}$ :

Pseudoemiliania lacunosa, ? Gephyrocapsa spp. Age: Probably Pseudoemiliania lacunosa Zone.
13-131A-1-2, 10-11 cm:

Age: Probably as above.

13-131A-3-1, 118-119 cm:

Pseudoemiliania lacunosa Zone.

13-131A-4-1, $110 \mathrm{~cm}$ :

Age: Probably as above.

13-131A-5-2, 4-5 cm:

Age: Probably as above.

13-132-1-1, 25-26 cm:

Gephyrocapsa spp., ?Emiliania huxleyi.

Age: Emiliania huxleyi Zone.

13-132-1-6, 6-7 cm:

Gephyrocapsa spp.

Age: Gephyrocapsa oceanica Zone.

13-132-2-1, $90 \mathrm{~cm}$ :

Age: As above.

13-132-2-4, 14-15 cm:

Age: As above.

13-132-3-1, 79-80 cm:

Age: As above.

13-132-3-4, 15-16 cm:

Pseudoemiliania lacunosa, Gephyrocapsa spp.

Age: Pseudoemiliania lacunosa Zone.

13-132-4-1, 119-120 cm:

Age: As above.

13-132-4-6, 20-21 cm:

Age: As above.

13-132-5-1, $10 \mathrm{~cm}$ :

Age: As above.

13-132-5-3, 10-11 cm:

Age: As above.

13-132-6-1, $16 \mathrm{~cm}$ :

Age: As above.

13-132-6-6, 30-32 cm:

Age: As above.

13-132-7-1, $9 \mathrm{~cm}$ :

Age: As above.

13-132-7-6, $5 \mathrm{~cm}$ :

Age: As above.

13-132-8-1, $15 \mathrm{~cm}$ :

Age: As above.

13-132-8-6, 5-6 cm:

Age: As above.

13-132-9-1, 10-11 cm:

Age: Probably oldest Pleistocene.

13-132-9-6, $10 \mathrm{~cm}$ :

Age: Pseudoemiliania lacunosa Zone.

13-132-10-1, $10 \mathrm{~cm}$ :

Age: As above.

13-132-10-6, $10 \mathrm{~cm}$ :

Discoaster brouweri; Discoaster pentaradiatus, Pseudoemiliania lacunosa.

Age: Discoaster brouweri Zone. 
13-132-11-1, $10 \mathrm{~cm}$ :

Age: As above.

13-132-11-4, $10 \mathrm{~cm}$ :

Discoaster variabilis, Discoaster surculus, Pseudoemiliania lacunosa .

Age: Discoaster surculus Zone.

13-132-12-1, $5 \mathrm{~cm}$ :

Age: As above.

13-132-12-2, $5 \mathrm{~cm}$ :

Age: As above.

13-132-13-1, $5 \mathrm{~cm}$ :

Discoaster asymmetricus, Discoaster tamalis, Discoaster surculus.

Age: Mid-Pliocene.

13-132-14-2, $10 \mathrm{~cm}$ :

Age: As above.

13-132-16-1, $5 \mathrm{~cm}$ :

Reticulofenestra pseudoumbilica, Sphenolithus abies, Discoaster pentaradiatus.

Age: Early Pliocene.

13-132-17-1, $5 \mathrm{~cm}$ :

Age: Probably as above (very poor preservation).

13-132-18-1, $5 \mathrm{~cm}$ :

Reticulofenestra pseudoumbilica, Discoaster brouweri.

Age: Late Miocene or early Pliocene.

13-132-19-2, $10 \mathrm{~cm}$ :

Discoaster brouweri, Discoaster pentaradiatus, cf. Discoaster brouweri rutellus, Reticulofenestra pseudoumbilica, Discoaster variabilis,

Age: Probably late Miocene.

13-132-20-2, $10 \mathrm{~cm}$ :

Age: As above.

13-132-21-2, $5 \mathrm{~cm}$ :

Age: As above.
13-132-22-1, $100 \mathrm{~cm}$ :

Age: Probably as above.

13-132-25-1, $05 \mathrm{~cm}$ :

Barren of nannofossils.

13-132-26-1, 13-14 cm:

Barren of nannofossils.

13-133-1-1, $130 \mathrm{~cm}$ :

Gephyrocapsa spp.

Age: Gephyrocapsa oceanica Zone.

13-133-1-2, $70 \mathrm{~cm}$ :

Age: As above.

13-133-5-1, $35 \mathrm{~cm}$ :

Barren of nannofossils.

13-134-3-1, $20 \mathrm{~cm}$ :

Discoaster variabilis, Pseudoemiliania lacunosa, Discoaster brouweri, Discoaster pentradiatus.

Age: Discoaster surculus Zone.

13-134-5-2, $31 \mathrm{~cm}$ :

Age: As above.

13-134-6-1, $20 \mathrm{~cm}$ :

Reticulofenestra pseudoumbilica, Sphenolithus abies, Discoaster brouweri, Discoaster asymmetricus.

Age: Early Pliocene.

13-134-7-1, $105 \mathrm{~cm}$ :

Age: As above.

13-134-7-5, 49-50 cm:

Age: Probably as above.

13-134A-1-1, $139 \mathrm{~cm}$ :

Gephyrocapsa spp.

Age: Gephyrocapsa oceanica Zone.

13-134A-1-2, $15 \mathrm{~cm}$ :

Age: As above. 